\title{
Testimonios indígenas conosureños: ¿convivencias excluyentes?
}

\author{
Southern Cone IndigenousTestimonies: Exclusive Coexistence?
}

\author{
HANS FERNÁNDEZ BENÍTEZ \\ Karl-Franzens-Universität Graz · hans.fernandez-benitez@uni-graz.at \\ Profesor Asistente de Literaturas Latinoamericanas en la Karl-Franzens-Universität Graz \\ (Austria). Estudió en la Universidad de Concepción (Chile) y se doctoró en la Humboldt- \\ Universität zu Berlin (Alemania). Entre sus áreas de investigación en la latinoamericanística \\ cuentan además de los estudios testimoniales, la relación entre literatura y prensa en el siglo XIX, \\ las literaturas indígenas y la investigación capuchina. Ha publicado la monografía De migrantes, \\ cuentistas, abigeos y cantores. El enfoque culturalista en los testimonios andinos «Gregorio \\ Condori Mamani» $y$ «Nosotros los humanos».
}

RECIBIDO: 26 DE OCTUBRE DE 2015

DOI: 10.7203/KAM.6.7194

ACEPTADO: 30 DE NOVIEMBRE DE 2015

ISSN: 2340-1869

Resumen: El presente artículo, partiendo de la premisa de que la teoría del testimonio en América Latina fue construida a partir de textos testimoniales del área centroamericana y caribeña sobre la base de un trasfondo marxista, revisa de manera panorámica testimonios de cuatro culturas indígenas de Sudamérica (quechua, aymara, mapuche y yagán) resaltando la dimensión cultural contenida en estos. Se caracterizan tanto las condiciones de producción de estos testimonios como también las formas de convivencia que estos representan. Las exclusiones que padecen los testimoniantes por parte de sus respectivas sociedades se interpretan como consecuencia de la idea ilustrada de progreso que guió la construcción de naciones en el siglo XIX. Pese a ello, los textos dan a conocer un Cono Sur polilógico. Palabras clave: testimonio, indígenas, Cono Sur, convivencia, exclusiones

\begin{abstract}
The present article begins with the premise that the theory of testimonio in Latin America was constructed based on testimonial texts from Central America and the Caribbean and with a Marxist underpinning. This article will examine the testimonies of four indigenous cultures of South America (Quechua, Aymara, Mapuche and Yaghan), highlighting the cultural dimension of each one, while at the same time characterizing the conditions of production as well as the social coexistence that they represent. The exclusions those who testify suffer in their respective societies are interpreted as a consequence of the Enlightenment idea of progress that guided nation building in the 19th Century. In spite of this, the texts illustrate a polylogical Southern Cone.
\end{abstract}

Key words: testimonio, indigenous peoples, Southern Cone, social coexistence, exclusions 


\section{Investigación testimonial latinoamericana y testimonios indígenas}

Considerando que la teoría del testimonio latinoamericano se elaboró principalmente a partir de los modelos centroamericano y caribeño Me llamo Rigoberta Menchú (Elisabeth Burgos y Rigoberta Menchú, 1983) y Biografía de un cimarrón (Miguel Barnet y Esteban Montejo 1966) en los cuales los estudios testimoniales latinoamericanos (Cf. Beverley y Achúgar 1992, Gugelberger 1996, Sklodowska 1992, entre otros) privilegiaron, a partir de la categoría de la "estética de la solidaridad", que ponía el acento en los vínculos supuestamente solidarios existentes entre el gestor y el testigo, el potencial marxista contenido en estos textos y que en 1996 John Beverley, uno de sus principales investigadores, declaró en vista de la crisis que afectaba a esta corriente investigativa: "The moment of testimonio is over" (280), el presente artículo analiza una constelación de testimonios prácticamente no tomada en cuenta por la crítica en los cuales se pone el acento en dimensiones relativas a las particularidades culturales de los testigos pertenecientes a sociedades vernáculas del Cono Sur y a la interacción de estos con la cultura dominante, vínculo en el cual se ven desfavorecidos, excluidos y finalmente condenados a la marginalidad.

Asimismo los investigadores del testimonio latinoamericano canonizaron a partir de las obras mencionadas la variante de la 'novela testimonial', excluyendo otras formas de expresión de este género en el continente, tal como el testimonio no novelado. Los testimonios conosureños que a continuación se revisan si bien presentan estilizaciones que corresponden a las huellas del trabajo editorial realizado por los gestores, despliegan más bien una intención antropológica en concordancia con su intención de rescate de las voces de las culturas autóctonas del Cono Sur.

La importancia de estos textos es central para enriquecer el canon testimonial latinoamericano, pues constituyen un corpus que puede contribuir a la elaboración de nuevas categorías de análisis en vista de sus especificidades estéticas, políticas y de sus respectivos contextos de producción-que permitan dar una visión más compleja del fenómeno de las prácticas testimoniales en Latinoamérica. Con este propósito, los testimonios que enseguida se analizan -y cuyos narradores pertenecen a sociedades autóctonas de distintas regiones culturales de Sudamérica- contribuyen a caracterizar la diversidad y riqueza de la producción testimonial del área conosureña.

Los cuatro textos considerados (Gregorio Condori Mamani. Autobiografía, Ricardo Valderrama y Carmen Escalante, 1977; Toribio Bartolo, Gastón Guzmán, 2009; Pascual Coña, Ernesto de Moesbach, 2006 y El zarpe final, Patricia Štambuk, 2007) corresponden a narrativas atravesadas por la dolorosa experiencia subjetiva de los procesos transculturales, pues sus narradores testimonian en el fondo acerca de distintas formas de interacción con los procesos de modernización y homogeneización cultural en sus respectivos países y contextos históricos. En vista de ello se observan a continuación los 'saberes de convivencia y de sobrevivencia' (Ette 2010) que estos testimonios indígenas conosureños representan así como las formas de negociar la convivencia que exponen. 


\section{Gregorio Condori Mamani}

El testimonio Gregorio Condori Mamani. Autobiografía contiene los relatos biográficos de Gregorio Condori y de su esposa Asunta Quispe, ambos monolingües en quechua e iletrados que migraron desde sus comunidades a la ciudad de Cusco. Los relatos fueron recogidos en el idioma de los testigos por la pareja de antropólogos peruanos Ricardo Valderrama y Carmen Escalante ${ }^{1}$ y publicados en edición bilingüe quechua-español en 1977, respecto a cuya traducción señalan: “El hecho de ser el runa simi nuestro idioma materno, ha facilitado que la traducción al castellano resulte lo más cercana posible al texto quechua, conservando así la forma muy peculiar de expresarse de los bilingües andinos de la zona" (Valderrama y Escalante, 1977: 15).

El origen de este testimonio - considerado, por lo demás, un clásico del contexto andino- se encuentra en el rodaje del documental El cargador (1974) del cineasta cusqueño Luis Figueroa, cortometraje que fue premiado en 1974 en el festival alemán de Oberhausen y que informa sobre las duras condiciones de vida que padecen los cargadores de soga en Cusco. Durante el proceso de realización del filme, Ricardo Valderrama se desempeñó como asistente antropológico.

Con respecto a la composición del libro, éste se acompaña de fotografías que muestran a los testigos en su casa y a Gregorio Condori cargando pesados bultos sobre su espalda a punto de ser aplastado. Estas expresivas imágenes pertenecen a Félix Nishiyama y a Eulogio Nishiyama, ambos, al igual que Luis Figueroa, integrantes del movimiento cinematográfico de la Escuela del Cusco.

Gregorio Condori al momento de las conversaciones con los antropólogos destinadas a recoger material para la elaboración del texto, se dedicaba al oficio de cargador en la ciudad de Cusco. Además de informar acerca de las características de su trabajo, Condori da a conocer en su testimonio un amplio repertorio de relatos míticos de la cultura quechua. En este sentido, cabe afirmar que Gregorio Condori es un auténtico narrador andino, cuyos mitos recogió durante sus desplazamientos por diferentes comunidades. Asunta Quispe, por su parte, se dedicaba a la venta de comida en las calles, y su testimonio profundiza en aspectos del género femenino de las mujeres migrantes.

Los gestores explican en la 'Nota Preliminar' que conocieron a los testigos cuando vivían en Coripata, uno de los barrios marginales de Cusco. En este lugar entablaron amistad con Gregorio Condori y Asunta Quispe, quienes eran sus vecinos. La relación de amistad y confianza con ellos se transformó en un pilar fundamental para la realización del testimonio, tal como señalan los gestores: "La amistad con Gregorio, su confianza en nosotros y sobre todo su asombrosa capacidad narrativa

\footnotetext{
${ }^{1}$ Valderrama y Escalante también publicaron el testimonio Nosotros los humanos (1992), texto que recoge las historias de vida, mitos y canciones de dos abigeos quechuahablantes, además de penetrar en el mundo de la violencia y de la religiosidad andinas. Otro testimonio quechua lo constituye Ciprian Phuturi Suni publicado en 1997 por Darío Espinoza.
} 
nos llevaron en 1975 a reanudar las entrevistas con él, dirigidas esta vez a reconstruir su historia de vida" (Valderrama y Escalante 1977: 13).

Vinculado con los antropólogos, Zevallos Aguilar (1998) explica que se trata de letrados biculturales que compartían la situación de pobreza con los testigos y que además el manejo del runa simi les permitió el acceso a información más profunda de la cultura quechua. Esta misma consideración permite comprender la capacidad de Valderrama y Escalante de haber sabido apreciar el dominio de Gregorio Condori del arte de narrar oralmente, respecto a cuyo talento verbal los antropólogos indican que éste demostraba admiración tanto por las habilidades narrativas de Matico (un cuentista que conoció en prisión) como por la oratoria del líder sindicalista Emiliano Huamantica (Cf. entrevistas en Fernández 2012). Las destrezas narrativas de Condori también quedan demostradas por medio de los procedimientos intertextuales que despliega a partir de un denso interdiscurso quechua al que recurre y por el hecho de que, como explica Cornejo Polar (1994), conecta su propia situación de alienación y dominación por no haber logrado la hispanización con la que padeció Atahuallpa, condenado a muerte por no saber leer.

Según Gregorio Condori y Asunta Quispe, su propósito al permitir publicar sus testimonios consiste en "que se conozcan los sufrimientos de los paisanos" (Valderrama y Escalante 1977: 15). Estos padecimientos al igual que el rico repertorio de mitos quechuas que Condori sacó a la luz no sólo fueron conocidos, sino que incluso el libro tuvo una resonancia positiva sobre los paisanos de los testigos, ya que sirvió de base para la realización de la Casa del cargador (instancia que ayuda a los migrantes serranos dedicados a este oficio) y mediante el testimonio de Quispe se alfabetizaron mujeres quechuas (Zevallos Aguilar, 1998).

Teniendo en cuenta que los testigos en 1977 vivían al lado de un basural, que nunca aprendieron español ni la lectoescritura y que sus condiciones de sobrevivencia eran durísimas, pues fueron condenados a una forma extrema de marginalidad, es imposible concebir una convivencia lograda en la representación que el testimonio ofrece del Perú de aquellos años. El texto representa más bien una exclusión de los testigos y de sus paisanos de la sociedad peruana, donde los quechuahablantes no participan en igualdad de condiciones y sólo se les otorga un lugar de subordinación. Por lo tanto, el testimonio Gregorio Condori Mamani no solamente rescata las voces de estos narradores que informan acerca de la historicidad de los quechuas contemporáneos y del discurso mítico de su cultura, sino que mediante las biografías articula una poderosa denuncia de la exclusión que sufren los testigos y sus paisanos por parte de una sociedad que no contribuyó a conseguir una convivencia, sino más bien los abandonó al no haberles otorgado la posibilidad de una integración -es decir, de una transculturación menos dolorosa- ni menos aún de ser respetados y valorados en su diferencia cultural. 


\section{Toribio Bartolo}

Toribio Bartolo. Testimonio de un aymara / Toribio Bartolo. Qamañapa (2009), editado en versión bilingüe aymara-español por Gastón Guzmán, corresponde al relato de vida del aymara Toribio Bartolo e incluye, además de fotografías relativas a su cultura, recuadros que contienen explicaciones culturales de conceptos andinos destinados a contextualizar y complementar los conocimientos del lector ${ }^{2}$.

Toribio Bartolo narra en su testimonio sobre sus desplazamientos por el mundo aymara en el marco de sus trabajos (en las salitreras, en el comercio), sobre su paso por el ejército, sobre aspectos de la religiosidad aymara, sobre su visión positiva de la educación chilena y sobre su retorno a su comunidad de origen.

Si bien Guzmán ha mencionado que el texto constituye parte de una investigación antropológica (2009: 11), considera como base del testimonio "[u]na larga permanencia en Chiapa entre los años 1980 y 1986 y una profunda relación de amistad establecida durante largo tiempo con don Toribio" (2009: 11). Al igual que en otros testimonios, el contacto prolongado con los testigos y su mundo representa un factor clave que permite a los gestores el acceso a información no sólo más personal, sino también más profunda de la cultura de los narradores.

De acuerdo con lo señalado por el editor, Toribio Bartolo manifiesta en su relato las estrategias empleadas por la cultura aymara para resistir a la aculturación:

Sus relatos nos hablan, en un lenguaje sencillo y franco, de las tradiciones de una sociedad que para sobrevivir a las influencias externas y a los cambios provenientes de las culturas dominantes, radicalmente opuestas a sus modos de vida, les han exigido transformar desde sus raíces, su pensamiento y su historia, sus estructuras de organización política, social y económica, para convivir con otras culturas y preservar su $<$ sic $>$ tradiciones, valores e identidad étnica (Guzmán, 2009: 13).

Por lo tanto, para la sociedad aymara asumir la transculturación implica, paradojalmente, la capacidad de adquirir estrategias que le permitan conservar elementos de la propia cultura en una forma negociada. Dicho de otro modo, la transculturación representa para el testigo -y su culturauna estrategia de sobrevivencia y de convivencia cultural, de hecho el capítulo sobre la escuela se acompaña de un recuadro que contiene una fotografía muy decidora de una niña que junto a su profesor en la sala de clases lee cuentos bilingües mientras en la pizarra se pueden ver los números en aymara y los días de la semana en inglés (Guzmán 2009: 107).

De acuerdo con lo señalado, el testimonio de Toribio Bartolo contiene una visión prospectiva de la cultura aymara, ya que será la transculturación la que permitirá la convivencia intercultural. Así, Guzmán escribe:

\footnotetext{
2 Otros testimonios de la cultura aymara que se pueden mencionar son 'Si me permiten hablar...'(1980), testimonio de Domitila Barrios editado por Moema Viezzer, y Manuela Ari (1995), editado por Lucy Briggs y Sabine Dedenbach.
} 
El testimonio de Don Toribio, que ha sido rigurosamente respetado y conservado, es una invitación a recorrer la historia de su pueblo y de una sociedad que se resiste a desaparecer, transformándose permanentemente para preservar su memoria y sus valores con la finalidad de continuar presentes en la construcción de un mundo que acepte y respete las tradiciones y los saberes culturales acumulados durante siglos (Guzmán, 2009: 14).

Por su parte, Lautaro Núñez Atencio describe en el prólogo -también en relación con la convivencia entre la cultura propia y la hegemónica- características del narrador en apariencia contradictorias: "Hombre que se sabe distinto por reconocerse "aymaristo", de esa generación de paisanos que rechazaban su indianidad” (Guzmán, 2009: 7) y simultáneamente "Don Toribio optó por los ideales de la ilustración educacional con que el Estado nacional aspiraba a homogenizar y chilenizar hasta los últimos rincones del país” (Guzmán, 2009: 8). Toribio Bartolo afirma la propia cultura, por ello quiere emplear los recursos que le ofrece la transculturación para evitar la extinción total del universo cultural aymara. Por lo tanto, para el testigo la sobrevivencia de su cultura es posible en base a una convivencia negociada con la sociedad dominante, ya que esta puede contribuir con los elementos indispensables que permitan preservar elementos propios y evitar así el colapso completo de su cultura.

\section{Pascual Coña}

Lonco Pascual Coña ñi tuculpazungun / Testimonio de un cacique mapuche (2006), publicado originalmente en $1930^{3}$, contiene en formato bilingüe mapudungun-español el relato de vida del mapuche Pascual Coña, textos etnográficos acerca de las particularidades de la cultura mapuche del siglo XIX, relatos ficticios y textos relativos a la visión mapuche de la naturaleza. Se trata de una obra enciclopédica que procura dar a conocer la cosmovisión mapuche y la organización cultural tradicional ${ }^{4}$.

El testimonio Pascual Coña surge en el contexto de la evangelización capuchina bávara del sur de Chile que tuvo lugar entre fines del siglo XIX e inicios del siglo XX $X^{5}$. El Estado chileno, tras la funesta y fallida intromisión del ejército en el territorio mapuche, consideró oportuna la intervención religiosa con la finalidad de civilizar, evangelizar y chilenizar a los mapuches. Los capuchinos

\footnotetext{
${ }^{3}$ Cabe destacar que el libro fue publicado originalmente con el título Vida y costumbres de los indigenas araucanos en la segunda mitad del siglo XIX. Foote (2012) ha estudiado en detalle los cambios de título y autor experimentados por este testimonio.

${ }^{4}$ Otros testimonios mapuches que se pueden mencionar son Sueño con menguante (1999) de Sonia Montecino y Una flor que renace (2002), testimonio de Rosa Reuque Paillalef editado por Florencia Mallon.

5 Pascual Coña demuestra que la existencia del testimonio en América Latina es más antigua que su consagración por parte de Casa de las Américas en 1970, como también consta en los trabajos etnográficos pioneros en materia testimonial llevados a cabo por Bernardino de Sahagún en el siglo XVI.
} 
alemanes llegaron al sur del país como consecuencia de un acuerdo establecido entre el Estado de Chile y el Vaticano.

Para llevar a cabo con éxito sus labores religiosos, los capuchinos requerían conocimientos de la lengua mapuche. En este contexto surgen gramáticas y diccionarios, uno de cuyos pioneros fue Félix José de Augusta, autor además de las Lecturas araucanas (1910), recopilación de relatos, cantos y tradiciones mapuches. Surge así una interesante producción filológica asociada a las misiones, contexto en el cual se ubica el testimonio Pascual Coña, el que constituye el resultado del dictado de Coña al religioso Ernesto de Moesbach.

En su testimonio Pascual Coña relata su vida dentro de su comunidad mapuche, su estadía en la misión capuchina donde aprendió a leer y escribir, su paso por la ciudad de Santiago donde también estudió y trabajó como carpintero, y finalmente el retorno a su comunidad de origen. Pascual Coña regresa al sur transformado en un mapuche chilenizado y manifiesta por ello serios problemas de readaptación. En estas circunstancias Coña dicta su testimonio a Moesbach.

Según declara el religioso en el prefacio con respecto a la elaboración del testimonio, "mi interés era al principio puramente lingüístico, [pues] [p]ara poder ejercer el ministerio sacerdotal entre los araucanos era indispensable aprender la lengua mapuche" (Moesbach, 2006: 22). Asimismo es importante tener en consideración el fundamento basado en la autenticidad e inteligencia de Coña que lleva a Moesbach a elegirlo como testimoniante, pues representa, según el capuchino, "un indígena legítimo de la antigua raza araucana, pero bastante instruido y dotado de una vida psíquica muy rica” (Moesbach 2006: 22).

Ernesto de Moesbach -al igual que sus hermanos de orden- estaba en contacto con el destacado mapuchista Rodolfo Lenz, quien escribe un entusiasta prólogo al testimonio destacando la prevalencia del punto de vista mapuche: "No he visto nunca una descripción tan detallada de costumbres sudamericanas, dada desde el punto de vista del indígena mismo" (Moesbach, 2006: 18). No obstante, Lenz defiende en este mismo texto una posición eurocéntrica ilustrada, según la cual los indígenas constituyen un lastre para el progreso de las sociedades modernas en América Latina, y así sobre esta base afirma que el testimonio expone "los problemas que ofrece su transformación [del indio] en un ciudadano útil” (Moesbach 2006: 19) y que al mismo tiempo el libro justifica la labor realizada por los capuchinos alemanes como encargo del Estado chileno: "La biografía de Coña nos muestra cómo se civiliza un indígena con la ayuda del clero" (Moesbach, 2006: 19). Dicho con otras palabras, Lenz únicamente acepta la cultura mapuche a un nivel de costumbres exóticas que, sin embargo, es preciso erradicar para conquistar el progreso definitivo del país de acuerdo con un concepto eurocéntrico de civilización.

Pascual Coña, por su parte, en su relato manifiesta ambigüedades respecto a su posicionamiento frente a su propia cultura, y así señala en su prólogo que los antiguos mapuches "tenían buenas costumbres, pero también malas" (Moesbach, 2006: 25), metaposición cultural derivada de su anterior convivencia con la sociedad chilena y con los capuchinos italianos y 
alemanes $^{6}$. Considerando esta afirmación tan crítica -e indicio de una aculturación si se refiere a la ritualidad mapuche-, cobra mayor sentido cuando precisa la programación de su relato, según la cual su vida transcultural representaría la superación de aquellas "malas costumbres" paganas: "contaré el desarrollo de mi propia existencia y también el modo de vivir de los antepasados" (Moesbach, 2006: 25). Pero enseguida, y teniendo en cuenta que él sale de la sociedad mapuche y luego procura reintegrarse, expresa el deseo de contribuir mediante la publicación de su testimonio en idioma mapudungun a la 'remapuchización' y a la preservación de su cultura de origen:

En nuestros días la vida ha cambiado; la generación nueva se ha chilenizado mucho; poco a poco ha ido olvidándose del designio y de la índole de nuestra raza; que pasen unos cuantos años y casi ni sabrán ya hablar su lengua nativa. Entonces, ique lean algunas veces siquiera este libro! (Moesbach, 2006: 25).

A partir de lo señalado, queda claro que Coña tuvo una vida muy agitada conviviendo en forma compleja con mapuches, italianos, chilenos y alemanes. Su testimonio narra en el fondo una reintegración fallida a su cultura de origen, puesto que según su relato es rechazado tanto por mapuches como por chilenos, y es aceptado por los alemanes quienes pretenden utilizar su testimonio como instrumento para llevar a cabo una evangelización más eficaz y así destruir la cultura mapuche tradicional. Se puede pensar que la vida Coña fue finalmente un producto de un experimento capuchino que consistió en transculturizar mapuches en aras del progreso.

El libro muestra, por lo demás, las contradicciones existentes entre los objetivos de Moesbach y de Coña con respecto a la publicación de la vida del narrador (Cf. Foote 2012). Para los capuchinos la vida de Coña es representativa del éxito de la evangelización, pero también se pueden considerar los alcances de ésta que condujo a los mapuches transculturados a una exclusión de la sociedad chilena. El hecho de que Pascual Coña dicte su testimonio a Moesbach en el refugio en que finalmente se transformó para él la misión Budi, es un claro indicio de la radicalidad de esta exclusión derivada de su condición transcultural.

En este sentido, cobra especial interés la crítica que Coña formula en su prólogo sobre sus paisanos que se han chilenizado más de la cuenta: quizás se trate también de una suerte de advertencia sobre las consecuencias negativas de esta transculturación, o bien represente el afecto que nunca perdió a la cultura mapuche, suposición que, sin embargo, se contradice con lo expresado en su epílogo, en el cual -muy probablemente a instancias de Moesbach- afirma, negando toda consideración positiva de su relato etnográfico y valorando paradojalmente los fundamentos de la transculturación de la cual fue víctima7, lo siguiente: "Ya han pasado las creencias deficientes de

\footnotetext{
${ }^{6}$ Previo a los capuchinos alemanes estuvieron los italianos y los españoles. Coña se educó con capuchinos italianos.

${ }^{7}$ Cabe suponer en vista de estas ambigüedades que tanto Coña como Moesbach quisieron mediante la publicación dejar un testimonio a los nuevos mapuches sobre cómo era la cultura mapuche tradicional previa a los procesos de transculturación que sufrió.
} 
nuestros antepasados, ya tenemos las enseñanzas que nos transmitió nuestro Señor. Es preciso aceptar con fe sus verdades y poner en práctica sus preceptos” (Moesbach, 2006: 485).

Por lo tanto, la biografía de Pascual Coña, pese a que este se movió entre diferentes culturas, es representativa de una convivencia fracasada y de dolorosas experiencias de exclusión. En su afán de chilenizarlo, los capuchinos lo marginalizaron doblemente: de la cultura mapuche y de la chilena. La civilización y el intento decimonónico de homogeneización cultural en aras del progreso condenó a Coña al aislamiento en la misión capuchina.

\section{Elzarpefinal}

El zarpe final. Memorias de los últimos yaganes (2007) corresponde a una recopilación monolingüe español de relatos acerca de la cultura yagán narrados por las hermanas Úrsula Calderón y Cristina Calderón, editados por Patricia Štambuk y complementados con fotografías, principalmente de retratos. Como se expresa en el texto de contraportada con respecto al contenido del libro, "[1]os relatos de ambas mujeres reconstruyen los mitos y leyendas de los yaganes y la existencia cotidiana de la comunidad en el transcurso del siglo XX” (Štambuk, 2007). La memoria de las narradoras funge como testimonio de la cotidianeidad remota de la cultura autóctona (ritos, mitos, relaciones humanas de diversa índole), especialmente a partir de la reconstrucción de retratos de integrantes de ésta.

En el prólogo, Štambuk indica que las hermanas Calderón nacieron en un tiempo "en que los padres ya no daban nombres indígenas a sus hijos" (Štambuk, 2007: 9), de hecho en el libro es frecuente la coexistencia de antropónimos yaganes con ingleses o españoles. Así, El zarpe final se compone de historias narradas desde el punto de vista de dos yaganes transculturadas y últimas hablantes nativas del idioma vernáculo ${ }^{8}$ que tematizan la vida cultural yagán antes y después del contacto con misioneros, colonos y chilenos. En este sentido, se puede señalar que el libro no sólo testimonia el proceso de extinción de una cultura, sino que se narra desde el punto de vista de miembros de esta que, junto con la editora, la perciben y presentan al borde de la desaparición total.

En relación con el origen del libro y con los propósitos de éste, Štambuk afirma lo siguiente:

Fue Rosa Yagán quien me embarcó por primera vez en la historia oral de su pueblo9 . Úrsula y Cristina, sus sobrinas lejanas, me permitieron continuar ese viaje por las costas meridionales de la Patagonia, para que sus memorias sobre los últimos yaganes de la Tierra del Fuego no mueran y pervivan en el acervo cultural chileno (2007: 9).

Por lo tanto, Štambuk concibe el testimonio como un poderoso instrumento de rescate de un patrimonio cultural en vías de desaparición, y si bien no da mayores indicaciones con respecto al

\footnotetext{
${ }^{8}$ Si bien existen descendientes, en la actualidad Cristina Calderón es la última hablante nativa del idioma yagán.

${ }^{9}$ En 1986 Štambuk publicó el libro Rosa Yagán.
} 
método de trabajo, al contexto de producción ni a las estilizaciones que efectuó en su calidad de editora ${ }^{10}$ y sólo cita entre corchetes si lo narrado proviene de Úrsula Calderón o Cristina Calderón, afirma la veracidad de los relatos que recrea: "Ellas me contaron los hechos verídicos que narro en este libro" (Štambuk 2007: 10).

La autoridad narrativa de las hermanas Calderón en cuanto testimoniantes se fundamenta en su conocimiento 'desde adentro' de la vida cultural de los yaganes, cuyo interdiscurso conocen de primera mano y retransmiten en sus relatos: "La orfandad temprana hizo que ambas pasaran la infancia y adolescencia yendo de familia en familia, de rancho en rancho, amparadas por los más viejos de la tribu. Así fue como escucharon y vieron lo que ahora relatan” (Štambuk 2007: 9).

Como fue señalado, el testimonio permite rescatar y registrar la cosmovisión de una cultura que se da por extinta. Asimismo informa sobre los procesos de transculturación sufridos por los yaganes que en la narración se manifiestan desde el nivel onomástico. El libro tematiza, basado en la memoria oral de las testigos transculturadas, la relación de los antiguos yaganes con la naturaleza, sus conocimientos sobre la vida y despliega una completa visión de mundo que ya no existe. De este modo, El zarpe final relata la desaparición de la cultura yagán como consecuencia del inevitable contacto cultural, es decir, narra la historia de la dramática exclusión de los yaganes de la sociedad chilena y de una convivencia que no ha sido posible.

\section{Convivencias que duelen}

Los testimonios revisados relatan situaciones de convivencias fallidas, de exclusiones y marginalizaciones de indígenas que han debido asumir la transculturación como forma de sobrevivencia en sus respectivos países. Los textos ponen en tela de juicio las consecuencias positivas de la Ilustración en América Latina, concretamente su proyecto de progreso en el Cono Sur. En el marco decimonónico de la construcción de naciones, el pensamiento ilustrado cumplió un papel destacado al contribuir con los fundamentos de un ideario que permitiría a los criollos construir naciones independientes del yugo español, sin precisar, no obstante, el rol que las sociedades autóctonas desempeñarían en tales proyectos, integrándolas sin más a los ideales criollos $^{11}$. La idea de la civilización, del progreso y de la construcción de naciones culturalmente homogéneas fue el trasfondo ideológico de la exclusión de los indígenas de las naciones modernas de América Latina.

\footnotetext{
${ }^{10} \mathrm{Al}$ inicio del libro, bajo una fotografía de ambas narradoras, aclara: "Las hermanas Úrsula (1925-2003) y Cristina (1928) Calderón Harban, informantes de las historias de este libro, en conversaciones ocurridas desde 1986 en la Patagonia Sur" (Štambuk 2007: 7).

11 Tanto para los investigadores del testimonio latinoamericano que canonizaron el género como para los criollos que guiaron los procesos de independencia en América Latina, los derechos civiles eran más importantes que los derechos culturales. Hay, sin duda, en ambas empresas un trasfondo de ideas ilustradas.
} 
Estos testimonios caracterizan la región conosureña no solo en lo que a su riqueza cultural se refiere, sino también en su conflictiva convivencia étnica. Se trata en los cuatro casos comentados de voces sobrevivientes de una modernidad que pretendió callarlas para siempre, por lo cual se puede afirmar que el testimonio opera como instrumento de rescate de éstas.

Pese a todo, aunque estos testimonios no forman parte del canon testimonial latinoamericano y ocupan una posición más bien marginal en las investigaciones testimoniales recientes, presentan un Cono Sur con abundancia de visiones de mundo. Así, este tipo de testimonio indígena de orientación antropológica permite captar otras lógicas culturales y, por tanto, representar un Cono Sur 'polilógico', consideración que permite ubicarlo dentro de las 'literaturas del mundo', de acuerdo con la formulación de Ottmar Ette:

Die Literaturen der Welt bieten als offene, vielsprachige und auf fundamental-komplexe Weise viellogische, polylogische Strukturierung weltweiten Zuschnitts nicht nur einen Erprobungsraum, sondern zugleich ein Reservoir an Wissensformen und Wissensnormen des Zusammenlebens (2012: VI) ${ }^{12}$.

Los testimonios indígenas conosureños al poner en primer plano la alteridad cultural de los testigos, despliegan, pues, todo su potencial estético y antropológico para captar y representar una región latinoamericana en su dimensión esencialmente polilógica. Los testimonios analizados contienen lógicas excluidas y subalternizadas por las repercusiones de una Ilustración homogeneizante en sus fundamentos progresistas que no las tomó en cuenta.

La transculturación es común a los cuatro textos, ya que otorga posibilidades de sobrevivencia. Se observa en estos testimonios una relación entre transculturación, resistencia y sobrevivencia. Las lógicas indígenas de Sudamérica son subsumidas al proyecto modernizador y la transculturación permite conservar parte de esta alteridad que se busca destruir. La transculturación, finalmente, favorece la sobrevivencia cultural.

De esta manera, es posible señalar que el proyecto ilustrado en América Latina fue de doble filo: por un lado, proporcionó las bases ideológicas para la conformación de los Estados nacionales, pero, por otro, sirvió de fundamento para llevar a cabo los genocidios o proyectos europeístas que aculturizaron o transculturizaron a las sociedades autóctonas del continente. Así, se puede pensar que estos testimonios de indígenas testimonian experiencias que resisten y sobreviven al proyecto modernizador de la Ilustración en el Cono Sur de América. Por lo demás, se trata de testimonios que a partir de la afirmación de la diferencia cultural de sus narradores y de la representación de un Cono Sur polilógico, se constituyen en narrativas críticas de la Ilustración en el contexto latinoamericano, la que proporcionó - como fue mencionado- los fundamentos en el siglo XIX para la construcción de sociedades culturalmente homogéneas y también ‘monológicas'. Los testigos logran convivir con la

12 Traducción propia: "En cuanto estructuración polilógica abierta a escala mundial, multilingüe y -de manera fundamentalmente compleja- multilógica, las literaturas del mundo ofrecen no sólo un espacio de experimentación, sino que al mismo tiempo un depósito de formas de conocimiento y normas de conocimiento de la convivencia”. 
sociedad dominante siempre y cuando se transculturicen, por lo cual las convivencias sólo son posibles en la medida en que éstos y su cultura no interfieran con su perturbadora diferencia en los proyectos de homogeneización cultural de las sociedades conosureñas. De este modo, considerando que los narradores son aceptados, pero no incluidos en el proyecto de sociedad, ya que su inclusión es incompatible con el concepto ilustrado de nación, los textos revisados testimonian en el fondo la existencia de posibles formas de convivencia fundamentalmente excluyentes. 


\section{Bibliografía citada}

Augusta, Félix José de (1910). Lecturas araucanas (narraciones, costumbres, cuentos, canciones, etc.). Valdivia: Imprenta de la Prefectura Apostólica (con la cooperación de Fray Sigifredo de Frauenhaeusl).

Barnet, Miguel ([1966] 2001). Biografía de un cimarrón. La Habana: Editorial Letras Cubanas.

Beverley, John (1996). “The Real Thing”. Gugelberger, Georg M. (ed.) The Real Thing. Testimonial Discourse and Latin America. Durham / London: Duke University Press: 266-286.

Beverley, John y Achugar, Hugo (directores) (1992). Revista de Crítica Literaria Latinoamericana. La voz del otro: testimonio, subalternidad y verdad narrativa. Año XVIII, $\mathrm{N}^{\circ} 36,2$ do Semestre. Lima / Pittsburgh: Latinoamericana Editores.

Briggs, Lucy T. y Dedenbach-Salazar Sáenz, Sabine (eds.) (1995). Manuela Ari: an aymara woman's testimony of her life (Text in Aymara, Englisch und Spanisch). Bonn: Bonner Amerikanistische Studien.

Burgos, Elisabeth (1983). Me llamo Rigoberta Menchú y así me nació la conciencia. La Habana: Casa de las Américas.

Cornejo Polar, Antonio (1994). Escribir en el aire. Ensayos sobre la heterogeneidad socio-cultural en las literaturas andinas. Lima: Editorial Horizonte.

Ette, Ottmar (2012). "Vorwort". Ette, Ottmar (Hrsg.) Wissensformen und Wissensnormen des Zusammenlebens. Literatur - Kultur - Geschichte - Medien. Berlin / Boston: Walter de Gruyter: V-VII.

Ette, Ottmar (2010). ZusammenLebensWissen. List, Last und Lust literarischer Konvivenz im globalen Maßstab (ÜberLebenswissen III). Berlin: Kulturverlag Kadmos.

Espinoza, Darío (recopilador) (1997). Testimonio. Ciprian Phuturi Suni. Tanteo puntun chaykuna valen. Las cosas valen cuando están en su punto de equilibrio.... Lima: CHIRAPAC.

Fernández, Hans (2012). De migrantes, cuentistas, abigeos y cantores. El enfoque culturalista en los testimonios andinos «Gregorio Condori Mamani» $y$ «Nosotros los humanos». Berlin: Logos Verlag.

Figueroa, Luis (1974). El cargador. Perú: Perucinex S. A.

Foote, Susan A. (2012). Pascual Coña: Historias de sobrevivientes. La voz en la letra y la letra en la voz. Concepción: Editorial Universidad de Concepción. 
Gugelberger, Georg M. (ed.) (1996). The Real Thing. Testimonial Discourse and Latin America. Durham / London: Duke University Press.

Guzmán G., Gastón (2009). Toribio Bartolo. Testimonio de un aymara / Toribio Bartolo Qamañapa. Santiago de Chile: Pehuén Editores.

Mallon, Florencia E. (edición y presentación) (2002). Una flor que renace. Autobiografía de una dirigente mapuche. Rosa Isolde Reuque Paillalef. Santiago de Chile: DIBAM / Centro de Investigación Barros Arana.

Moesbach, Ernesto Wilhelm de. ([1930] 2006). Lonco Pascual Coña ñi tuculpazugun. Testimonio de un cacique mapuche. Santiago de Chile: Pehuén Editores.

Montecino, Sonia (1999). Sueño con menguante. Biografía de una machi. Santiago de Chile: Editorial Sudamericana.

Sklodowska, Elzbieta (1992). Testimonio hispanoamericano. Historia, teoría, poética. New York / San Francisco / Bern / Baltimore / Frankfurt am Main / Berlin / Wien / Paris: Peter Lang.

Štambuk M., Patricia (2007). El zarpe final. Memorias de los últimos yaganes. Santiago de Chile: LOM Ediciones.

Štambuk M., Patricia (1986). Rosa Yagán, el último eslabón. Santiago de Chile: Editorial Andrés Bello.

Valderrama Fernández, Ricardo y Escalante Gutiérrez, Carmen (1992). Nosotros los humanos. Nuqanchik Runakuna. Testimonios de los quechuas del siglo XX. Cusco: Centro de Estudios Regionales Andinos “Bartolomé de las Casas”.

Valderrama Fernández, Ricardo y Escalante Gutiérrez, Carmen (1977). Gregorio Condori Mamani. Autobiografía. Cusco: Centro de Estudios Rurales Andinos “Bartolomé de las Casas”.

Viezzer, Moema ([1977] 1980). 'Si me permiten hablar...'Testimonio de Domitila, una mujer de las minas de Bolivia. México: Siglo XXI.

Zevallos Aguilar, Juan. “A propósito de Andean Lives, Gregorio Condori Mamani y Asunta Quispe Huamán. Apuntes sobre la hipercanonización y negligencias de la crítica del testimonio". Revista de crítica literaria latinoamericana. Año XXIV, N 48 (1998): 241-248. 